\section{Advocating for the consumer: clinical confirmation of all direct- to-consumer raw data alterations remains critical}

Wu et al. ${ }^{4}$ offer comments on our article in which we evaluate the quality and false positive rate of consumer obtained raw data files from direct-to-consumer (DTC) testing and the use of third-party interpretation services to decipher those files. As stated by $\mathrm{Wu}$ et al., our study was not examining the health-related reports that a consumer is provided by 23andMe when purchasing their product. Therefore the Food and Drug Administration (FDA) approval of a handful of health-related single-nucleotide polymorphisms (SNPs) (out of the hundreds of thousands of SNPs captured by their platform) is not the basis of our analysis. Our intent was to provide evidence for medical professionals and the general public who may not have a strong genetics background that caution should be taken when requesting raw data from a DTC company. Specifically, there is a potential for false positive results as well as variant classification discrepancies with use of third-party interpretation services. ${ }^{1}$

$\mathrm{Wu}$ et al. state that if not for DTC testing, many people in our cohort with confirmed results may not have ever learned about this important health knowledge. We queried the six FDA-approved SNP alterations in our cohort that Wu et al. would have reported on: the three BRCA1/2 Ashkenazi Jewish founder mutations $(n=13)$, the one CFTR founder mutation $(n=4)$, and the two familial Mediterranean fever founder mutations $(n=1)$. While these FDA-approved SNPs were all clinically confirmed as expected, these accounted for less than half of individuals in this study seeking confirmatory testing $(n=18 / 49,37 \%)$.

The remaining $31 / 49$ (63\%) individuals had findings that 23andMe does not report and therefore they would not have learned this important health information unless they requested their raw data. In our study, $47 / 49$ cases were from 23andMe. Of these 47 individuals, 18 (38\%) had confirmed FDA-approved pathogenic variants in BRCA1/2, CFTR, or $M E F V$. Seven (15\%) received true calls in non-FDA approved alterations. The remaining 22 individuals (47\%) were found to have false positives from the raw data from 23 andMe. ${ }^{1}$

While we agree with $\mathrm{Wu}$ et al. in that an individual has a right to their personal genetic information and their raw data, we feel strongly that an individual also has a right to know all of the caveats and limitations that go along with this data. Even though the raw data obtained from 23andMe is accompanied by a disclaimer that the information is neither validated for accuracy nor intended for medical use, these warnings and disclaimers are not always heeded or fully understood by the consumer and some medical providers. Therefore we stress the importance of taking that data (the raw data or a third-party interpretation report) to a medical provider for clinical confirmation before taking any actions related to health. As stated by Dr. M.J. Friez, "That said, consumers expect the quality of the data in question, no matter its origin, to be accurate-what they decide to do with it is their own personal business....Moreover, while the DTC community is convinced that they are doing all of us a favor with this medical model, there are many elsewhere that are not even remotely confident that this is the most appropriate method to disseminate information with potentially important medical consequences." ${ }^{2}$ Perhaps 23andMe would consider expanding their current disclaimer to include more detailed information about utilizing a consumer's clinical history and to further stress the need for clinical confirmation of findings prior to medical use.

We would also like to clarify several statements made by $\mathrm{Wu}$ et al. First, they stated that " 26 variants (a majority of the findings studied) were confirmed to be accurate." We confirmed 10 unique variants a total of 26 times, as there were multiple confirmation requests for some variants. Likewise, we observed 13 unique variants that were found to be false positives a total of 17 times. Importantly, the CHEK2 c.1100delC founder mutation was confirmed in two individuals but also found to be a false positive in two individuals. Second, $\mathrm{Wu}$ et al. claimed that we cited differences in testing technologies between DTC and clinical labs as an explanation for the high false positive rates; however, this is incorrect as any raw data files, whether resulting from sequencing or genotyping, will have inherent inaccuracies. It is important for consumers and medical providers to appreciate the likelihood of the inaccuracies with a raw data file. We feel that 23andMe's commentary about the technological differences is misguided. Third, $\mathrm{Wu}$ et al. claimed there were "variants that Ambry did not have the technology to test." They were referring to the four COL5A1 variants (Table 3 ), which we did not analyze due to their deep intronic location, which is out of standard reporting range for all clinical diagnostic labs, and because these variants are not deemed medically relevant findings by any clinical lab. Ambry's technology was not a factor in this scenario.

Despite the small sample size and ascertainment bias of our study (as mentioned in our manuscript), it is the first to investigate the caveats of requesting raw data and the use of third-party interpretation services. We encourage additional diagnostic laboratories and independent researchers to 
investigate the concerns we have raised. As DTC genetic testing gains popularity, there is a greater need to continue to educate the public and medical professionals about its limitations and caveats. In addition, a testing laboratory of any kind needs to take responsibility in how genetic information is being disseminated. ${ }^{3}$ Laboratories, the medical community, and the public all need to work together to responsibly handle genetic information. Several national professional organizations such as the American College of Medical Genetics and Genomics (ACMG), American Society of Human Genetics (ASHG), National Society of Genetic Counselors (NSGC), and the Centers for Disease Control and Prevention (CDC) have supported this concept through policy statements regarding the use of DTC testing. Genetics professionals and nongenetics medical professionals must collaborate to assure that quality genetic information is being provided, as this impacts the perception of the entire genetics field.

\section{DISCLOSURE}

All authors except for Jenna Guiltinan are full-time employees of Ambry Genetics. Jenna Guiltinan is currently a full time employee of Kaiser Permanente. Otherwise there are no conflicts of interest to declare from any of the authors.
Stephany Tandy-Connor, $M S^{1}$, Kate Krempely, $M S^{1}$, Tina Pesaran, $M S^{1}$, Holly LaDuca, $M S^{1}$, Jenna Guiltinan, $M S^{1}$ and

Brigette Tippin Davis, PhD, FACMG ${ }^{1}$

${ }^{1}$ Ambry Genetics, 15 Argonaut, Aliso Viejo, CA 92656, USA. Correspondence: Stephany Tandy-Connor (sconnor@ambrygen.com)

\section{REFERENCES}

1. Tandy-Connor S, Guiltinan J, Krempely K, et al. False-positive results released by direct-to-consumer genetic tests highlight the importance of clinical confirmation testing for appropriate patient care [published online ahead of print March 22, 2018]. Genet Med. https://doi.org/10.1038/ gim.2018.38.

2. Friez MJ Attention: direct-to-consumer patrons: proceed with caution [published online ahead of print April 30, 2018]. Genet Med. https://doi. org/10.1038/s41436-018-0030-8.

3. Ramos E, Weissman S. The dawn of consumer-directed testing. Am J Med Genet Sem Med Genet. 2018;178:89-97.

4. Wu S, Pollard J, Chowdry A, et al. Genet Med. 2018 [Epub ahead of print]

Advance online publication $\mathrm{xx}$ xx 2018. doi:10.1038/s41436-018-0095-4 